\title{
Diseño de un modelo de programación lineal para la planeación de producción en un cultivo de fresa, según factores costo/beneficio y capacidades productivas en un periodo temporal definido
}

\author{
Daniel Sánchez Pineda \\ Docente - Programa de Ingeniería Industrial \\ Universidad Militar Nueva Granada \\ tmp.daniel.sanchez@unimilitar.edu.co
}

\author{
Natalia Ramírez Torres \\ Docente - Programa de Ingeniería Industrial \\ Universidad Militar Nueva Granada \\ natalia.ramirez@unimilitar.edu.co
}

(Tipo de Artículo: Investigación Científica y Tecnológica. Recibido el 12/07/2016. Aprobado el 07/09/2016)

\begin{abstract}
Resumen. El sector hortifrutícola es considerado como una de las vías de ingreso de la población colombiana. Actualmente el departamento de Cundinamarca se destaca como el mayor productor de fresa, con una participación del $63,4 \%$ en la producción nacional. La fresa, conocida en el ámbito científico como fragaria vesca, presenta alto consumo gracias a las cualidades de aroma, color y acidez que presenta. La planta de la fresa, se caracteriza por ser perenne, al formar nuevos tallos de forma constante. Un cultivo de fresa, presenta una duración promedio de dos años, seccionados en etapas de funcionamiento. Son escazas las metodologías, que permitan generar una planeación de la producción a nivel frutícola, teniendo en cuenta el comportamiento colombiano. Es por ello que el estudio desarrollado incluye una propuesta de diseño para la planeación de la producción de fresa; teniendo en cuenta factores de costo/beneficio en un cultivo; a partir de programación lineal. Se muestra la formulación matemática y una verificación teórica de su funcionamiento y se evidencia el modelo a nivel teórico es funcional, permitiendo establecer un sistema de verificación de capacidades productivas, de acuerdo a parámetros de entrada específicos.
\end{abstract}

Palabras clave. Capacidades productivas, costo/beneficio, cultivo de fresa, modelo de programación lineal, planeación de producción.

\section{Linear programming model design for production planning in a strawberry crop, according to cost / benefit factor and productive capacity in a temporary period defined}

\begin{abstract}
Today, the fruit and vegetable sector is considered as one of the main entry routes for much of the Colombian population. Among the featured products, the strawberry production level in Colombia, has domestic consumption and industrial processing as the main destination. Currently the department of Cundinamarca stands out as the largest producer of strawberry, with a share of $63.4 \%$ in domestic production. Strawberry, known in the scientific area as Fragaria vesca, has high consumption thanks to the qualities of aroma, color and acidity that presents. The strawberry plant is characterized as being perennial, to form new stems steadily. A strawberry crop, has an average duration of two years, sectioned operating stages, the first stage known as stage vegetative character, includes the development of plants planted, the second stage is the first production stage of the crop, followed by a stage of pruning and renewal. The fourth and fifth stage corresponding to the second production stage and pruning and final renewal of useful cultivation period. From the exposed production conditions, they are scarce methodologies that allow generating a production planning for fruit and vegetable sector, taking into account the Colombian behavior. That is why the study developed includes a design proposal for planning strawberry production; taking into account factors of cost / benefit in culture; from linear programming. The mathematical formulation and theoretical verification of performance is shown and the evidence that the theoretical model is functional, allowing to establish a system for production capacity verification, according to specific input parameters.
\end{abstract}

Keywords. Cost/benefit, linear programming model, production planning, productive capacity, strawberry crop. 


\section{Introducción}

Hoy en día el sector hortifrutícola se considera como una de las principales vías de ingreso para gran parte de la población colombiana. Entre los productos destacados, la fresa a nivel productivo en Colombia, tiene como destino principal el consumo nacional y la transformación industrial. En el año 2011 se registró un total de 1135 hectáreas cultivadas, que en proporción representaron una producción aproximada de 45203 t. Actualmente el departamento de Cundinamarca se destaca como el mayor productor de fresa, con una participación del $63,4 \%$ en la producción nacional. El plan frutícola nacional, incluye una propuesta del incremento de las áreas sembradas para la producción de fresa, en un $380 \%$ con el fin de generar niveles de exportación. La fresa, conocida en el ámbito científico como fragaria vesca, presenta alto consumo gracias a las cualidades de aroma, color y acidez que presenta. La planta de la fresa, se caracteriza por ser perenne, al formar nuevos tallos de forma constante. Su crecimiento, se encuentra sujeto a las condiciones de luz y temperatura, por lo que se estima que la altitud adecuada para su producción se establece entre 1300 y $2000 \mathrm{~m}$. Germina en suelos profundos francos o franco arenosos, manteniendo un pH entre 6 y 7 unidades. Debe ubicarse en los primeros $15 \mathrm{~cm}$ del suelo. Un cultivo de fresa, presenta una duración promedio de 2 años, seccionados en etapas de funcionamiento, la primera etapa conocida como etapa de carácter vegetativo, cuya duración promedio es de 4 a 6 meses; de acuerdo al tipo de fragaria a cultivar; incluye el desarrollo de las plantas sembradas. La siembra se recomienda a través del proceso a través de macrotúneles, teniendo en cuenta que ha sido implementado en sistemas productivos tecnificados, generando ventajas como la reducción de la generación de lixiviados, provenientes de los fertilizantes, así como en la inversión realizada en pesticidas y el control de incremento de la temperatura $[1,2]$.

La segunda etapa denominada como etapa productiva, con una duración aproximada de 7 meses, corresponde a la producción del falso fruto para recolección, donde se indica que, por planta sembrada, se generarán en promedio $1,4 \mathrm{~kg}$ de fresas. La primera sesión productiva se cataloga como la de mayor rendimiento en el cultivo. Posteriormente la tercera etapa corresponde a la poda y renovación de cultivo; con una duración promedio de 2 meses; que se incluye como un paso requerido de mantenimiento en el cultivo, que permite verificar las condiciones de las plantas. La cuarta etapa, corresponde a la segunda sesión de producción; cuya duración es de 7 meses de igual forma; donde la producción promedio por planta disminuye a $0,6 \mathrm{~kg}$ de fresas por planta sembrada. Finalmente se realiza una poda y renovación para finalizar el periodo útil del cultivo $[3,4]$.

A partir de las condiciones productivas expuestas, son escazas las metodologías, que permitan generar una planeación de la producción a nivel frutícola, teniendo en cuenta el comportamiento colombiano. Es por ello que el estudio desarrollado incluye una propuesta de diseño para la planeación de la producción de fresa; teniendo en cuenta factores de costo/beneficio en un cultivo; a partir de programación lineal, con el objetivo de identificar capacidades productivas respecto a las etapas de cultivo y su relación con la sostenibilidad esperada. Se muestra la formulación matemática y una verificación teórica de su funcionamiento. El presente artículo surge como producto derivado del proyecto de investigación INV_ING_1915 de la Universidad Militar Nueva Granada.

\section{Estado del arte}

Tras la introducción, se presentan los aspectos relevantes asociados a la producción de fresa, desde sus características, al condicionamiento en el territorio colombiano.

\subsection{Características en la producción de fresa}

La fresa, conocida en el ámbito científico como fragaria vesca, presenta alto consumo a nivel mundial, gracias a las cualidades de aroma, color y acidez que presenta. La planta de la fresa, se caracteriza por ser perenne, al formar nuevos tallos de forma constante. Su crecimiento, se encuentra sujeto a las condiciones de luz y temperatura, por lo que se estima que la altitud adecuada para su producción se establece entre 1300 y $2000 \mathrm{~m}$. Germina en suelos profundos francos o franco arenosos, manteniendo un $\mathrm{pH}$ entre 6 y 7 unidades. Debe ubicarse en los primeros $15 \mathrm{~cm}$ del suelo. Adicionalmente, previo al proceso de producción, debe llevarse a cabo la etapa de desinfección, para mitigar la presencia de hongos, ácaros, hierbas, parásitos e insectos. Su producción, puede efectuarse de dos formas, a campo abierto o macrotúneles $[5,6]$.

La producción a campo abierto, presenta una gran desventaja, teniendo en cuenta que el cultivo cuenta con limitaciones bióticas, por lo que se encuentra expuesto a pueden presentarse plagas, ácaros y larvas. Adicionalmente, en relación a las restricciones abióticas, las condiciones climáticas adversas, los vientos, heladas y altas precipitaciones [7].

El proceso a través de macrotúneles, ha sido implementado en sistemas productivos tecnificados, generando ventajas como la reducción de la generación de lixiviados, provenientes de los fertilizantes, así como en la inversión realizada en pesticidas, incremento de la temperatura. El sistema agronómico característico de este proceso, se compone de tres etapas principalmente: vivero, instalación del cultivo, mantenimiento y cosecha. Inicialmente, se implementan eras con un ancho entre 70 y $80 \mathrm{~cm}$, de altura $20 \mathrm{~cm}$. En cada una de las eras se procede a implementar dos hileras, separadas $40 \mathrm{~cm}$ entre ellas. Posteriormente, las eras deben ser cubiertas con polietileno de color negro, cuya característica de grosor varía entre 0,2 a 0,4 mm; estableciendo un aislamiento del suelo, reduciendo de esta forma los problemas fitosanitarios, crecimiento de 
maleza y aumentando la retención de humedad y temperatura $[8,9]$.

Los sistemas de riego, pueden ser por aspersión o por goteo, siendo este último el de mayor implementación, manteniendo salidas de agua cada $25 \mathrm{~cm}$. Después de su recolección, las fresas deben ubicarse en cámaras frías, considerando que, a temperatura ambiente, se deteriora $80 \%$ en 8 horas. El proceso de selección, se encuentra condicionado al tamaño, grado de maduración, uniformidad y nivel de sanidad del fruto [10].

\section{Métodos y materiales}

El estudio derivado del proyecto de investigación INV_ING_1915, de la facultad de ingeniería de la Universidad Militar Nueva Granada - UMNG sede Calle 100 , se realizó con el objetivo de diseñar un modelo de programación lineal, cuyo objetivo es la identificación de capacidades productivas respecto a las etapas de cultivo y su relación con la sostenibilidad esperada en un sistema de tipo macrotúneles. Este modelo se estableció a partir de fuentes de información secundaria, relacionada al comportamiento del cultivo a nivel Colombia, orientado específicamente al departamento de Cundinamarca.

El diseño del modelo, se basa en el análisis productivo de cultivo de fresa, cuya duración promedio respecto a vida útil es de 2 años; a pesar de que se afirma que es un cultivo perenne; seccionados en etapas de funcionamiento. La primera etapa conocida como etapa de carácter vegetativo, cuya duración promedio es de 4 a 6 meses; de acuerdo al tipo de fragaria a cultivar; incluye el desarrollo de las plantas sembradas, donde se realiza la adecuación del terreno, ubicación de sistemas de riego y construcción de las hileras de macrotúneles. El sistema de macrotúneles es recomendado teniendo en cuenta que ha sido implementado en sistemas productivos tecnificados, permitiendo generar ventajas como la reducción de la generación de lixiviados, provenientes de los fertilizantes, así como en la inversión realizada en pesticidas y el control de incremento de la temperatura. Para la siembra se establece un promedio de ubicación de 4 plantas por cada metro cuadrado disponible en una hilera de macrotúnel [3].

La segunda etapa denominada como etapa productiva, cuya duración aproximada de 7 meses en terreno colombiano, corresponde a la producción del falso fruto para recolección, donde se indica que, por planta sembrada, se generarán en promedio $1,4 \mathrm{~kg}$ de fresas, durante el total del primer periodo productivo. La primera sesión productiva se cataloga como la de mayor rendimiento en el cultivo; definiendo que durante los primeros 3 meses se genera el $60 \%$ de la producción, durante los 2 meses posteriores el $25 \%$ y en los 2 meses finales el $15 \%$ de la producción. Posteriormente la tercera etapa corresponde a la poda y renovación de cultivo; con una duración promedio de 2 meses; que se incluye como un paso requerido de mantenimiento en el cultivo, que permite verificar las condiciones de las plantas, realizar la limpieza y retiro de plantas no requeridas, el control de plagas y la verificación del estado de los sistemas de riego y macrotúneles. La cuarta etapa, corresponde a la segunda sesión de producción; cuya duración es de 7 meses de igual forma; donde la producción promedio por planta disminuye a $0,6 \mathrm{~kg}$ de fresas por planta sembrada, manteniendo el mismo comportamiento porcentual, respecto a la primera etapa. Finalmente se realiza una poda y renovación para finalizar el periodo útil del cultivo $[11,3]$.

Teniendo en cuenta las condiciones previamente descritas, a continuación, se presenta la descripción de la construcción del modelo [12].

\subsection{Diseño del modelo}

A continuación se presenta la primera sección del modelo correspondiente a los parámetros de ingreso, donde se debe definir el área disponible de cultivo en metros cuadrados, el precio de venta promedio por $\mathrm{kg}$ de fresa, la producción promedio de $\mathrm{kg}$ de fresa por planta en cada una de las etapas productivas de ecuaciones, el número de plantas a sembrar en cada 10000 metros cuadrados; o plantas por metro cuadrado; y los valores de costo de establecimiento; correspondiente a las actividades de construcción de los macrotúneles, contemplando costos de material y operativos; y costo de mantenimiento; asociado a las labores diarias de verificación; ambos valores contemplados en el periodo funcional del cultivo, correspondiente a dos años.

Parámetros de entrada

$A_{T}=$ Área disponible para cultivo en metros cuadrados en un periodo de 2 años

$P V_{u}=$ Precio de venta por unidad $(\mathrm{kg})$ de fresa

$T P_{e 1}=$ Tasa de producción de $\mathrm{kg}$ de fresa por planta durante la etapa productiva 1

$T P_{e 2}=$ Tasa de producción de $\mathrm{kg}$ de fresa por planta durante la etapa productiva 2

$D P=$ Densidad de plantas sembradas por

metro cuadrado

$C E_{P}=$ Costo de establecimiento por planta

durante un periodo de planeación de 2 años

$C M_{P}=$ Costo de mantenimiento por planta

durante un periodo de planeación de 2 años

Las variables del modelo, orientadas a la definición de las cantidades de $\mathrm{kg}$ de fresa a recolectar, que dependerán de las definiciones de capacidad productiva del cultivo.

Variables de decisión

$X_{i j}=$ Cantidad de $\mathrm{kg}$ de fresa recolectados en etapa $i$ para el mes $j$

$$
i=\left\{\begin{array}{c}
1 \text { Etapa vegetativa } \\
2 \text { Etapa productiva } 1 \\
3 \text { Etapa de poda y renovación } 1 \\
4 \text { Etapa productiva } 2 \\
5 \text { Etapa de poda y renovación } 2
\end{array}\right.
$$




$$
j\left\{\begin{array}{c}
\operatorname{para} i=1 \ldots j=1, \ldots, 6 \\
\operatorname{para} i=2 \ldots j=1, \ldots, 7 \\
\operatorname{para} i=3 \ldots j=1,2 \\
\operatorname{para} i=4 \ldots j=1, \ldots, 7 \\
\operatorname{para} i=5 \ldots j=1,2
\end{array}\right.
$$

La función objetivo; ecuación 1; se asocia a la maximización de la utilidad del cultivo, relacionada a las ventas esperadas del total de $\mathrm{kg}$ recolectados de fresa durante las etapas productivas y los costos totales de establecimiento y mantenimiento, relacionados con el total de plantas sembradas.

Función Objetivo

$$
\begin{aligned}
\operatorname{MAX} Z=\sum_{j=1}^{7} X_{2 J} * P V_{u}+\sum_{j=1}^{7} X_{4 J} * P V_{u} \\
-\left(D P * A_{T} * C E_{P}+D P * A_{T} * C M_{P}\right)
\end{aligned}
$$

La función dependerá de 5 bloques de restricciones, el primero asociado a la producción en etapa vegetativa, teniendo en cuenta que no se genera ningún fruto durante los primeros 6 meses de producción. El segundo bloque, corresponde a la producción en las etapas de poda y renovación, teniendo en cuenta que en dichas etapas tampoco se genera producción. El tercer y cuarto bloque; ecuaciones 2 a 17; corresponden a las etapas productivas 1 y 2 respectivamente, donde la cantidad de $\mathrm{kg}$ de fresa a recolectar por mes, dependerá directamente de la capacidad definida de producción mensual, relacionada con el área disponible, el número de plantas sembradas y la producción esperada por planta, respecto al rendimiento. Se incluye adicionalmente una restricción de control de costos, donde se define que la sumatoria de las ventas esperadas de la primera etapa productiva, deben cubrir al menos un 59\% del total de los costos correspondientes a los 2 años del cultivo. De la misma manera las ventas esperadas de la segunda etapa productiva, deben cubrir al menos un $49 \%$ de los costos totales. Esto se relaciona a los cambios de rendimiento presentes en las etapas.

Bloque de restricciones etapa vegetativa

$X_{1 j}=0 \quad \forall j=1, \ldots, 6$

Bloque de restricciones etapas de poda y renovación

$X_{i j}=0 \quad \forall i=3,5 \quad \forall j=1,2$

Bloque de restricciones etapa productiva 1

$X_{21} \leq 18 \% * D P * A_{T} * T P_{e 1}$

$X_{22} \leq 24 \% * D P * A_{T} * T P_{e 1}$

$X_{23} \leq 18 \% * D P * A_{T} * T P_{e 1}$

$X_{24} \leq 17 \% * D P * A_{T} * T P_{e 1}$

$X_{25} \leq 8 \% * D P * A_{T} * T P_{e 1}$

$X_{26} \leq 8 \% * D P * A_{T} * T P_{e 1}$

$X_{27} \leq 7 \% * D P * A_{T} * T P_{e 1}$

$\sum_{j=1}^{7} X_{2 J} * P V_{u} \geq 59 \% *\left(D P * A_{T} * C E_{P}+D P * A_{T} *\right.$

$\left.C M_{P}\right)$

Bloque de restricciones etapa productiva 2

$X_{41} \leq 18 \% * D P * A_{T} * T P_{e 2}$

$X_{42} \leq 24 \% * D P * A_{T} * T P_{e 2}$
Ingenierías USBMed, Vol. 8, No. 1, Enero-Junio 2017

$X_{43} \leq 18 \% * D P * A_{T} * T P_{e 2}$

$X_{44} \leq 17 \% * D P * A_{T} * T P_{e 2}$

$X_{45} \leq 8 \% * D P * A_{T} * T P_{e 2}$

$X_{46} \leq 8 \% * D P * A_{T} * T P_{e 2}$

$X_{47} \leq 7 \% * D P * A_{T} * T P_{e 2}$

$\sum_{j=1}^{7} X_{4 J} * P V_{u} \geq 41 \% *\left(D P * A_{T} * C E_{P}+D P * A_{T} *\right.$

$\left.C M_{P}\right)$

El quinto bloque corresponde a la condición de no Negatividad presente en el sistema, para las variables de decisión.

No Negatividad

$X_{i j} \geq 0 \quad \forall i, j$

\section{Resultados}

A partir del modelamiento planteado, se realiza una prueba de verificación del funcionamiento teórico del mismo, comprobando los resultados con el software GAMS. En la Tabla 1 se presentan los parámetros definidos para el caso.

A partir de los parámetros, se realiza la ejecución del modelo, cuyos resultados se pueden evidenciar en la Tabla 2.

A partir de los resultados evidenciados, se presenta el total de los costos y la maximización de la utilidad en la Tabla 3, de acuerdo al modelo.

Se evidencia, que el modelo a nivel teórico es funcional, permitiendo establecer un sistema de verificación de capacidades productivas de un cultivo de una hectárea, para 40.000 plantas sembradas, donde; con el supuesto de recolección en cada mes del total de la capacidad productiva, como ventas esperadas totales; se logra generar una utilidad del cultivo, en los 2 años de periodo de planeación, de \$ COP 72.000.0000.

Tabla 1. Parámetros para el modelo. Fuente: Autores

\begin{tabular}{lc}
\hline Área disponible $\left(\mathrm{m}^{\wedge} 2\right) A_{T}$ & $10.000,00$ \\
\hline $\begin{array}{l}\text { Precio de venta promedio } \\
\text { por kg } P V_{u}\end{array}$ & $\$ 1.800,00$ \\
\hline $\begin{array}{l}\text { Producción kg/planta etapa } \\
1 T P_{e 1}\end{array}$ & 1,40 \\
\hline $\begin{array}{l}\text { Producción kg/planta etapa } \\
2 T P_{e 2}\end{array}$ & 0,60 \\
\hline $\begin{array}{l}\text { Plantas por cada 10.000 } \\
\mathrm{m}^{\wedge} 2 D P\end{array}$ & $40.000,00$ \\
\hline $\begin{array}{l}\text { Costo de } \\
\text { establecimiento/planta } C E_{P}\end{array}$ & $1.000,00$ \\
\hline $\begin{array}{l}\text { desto } \\
\text { mantenimiento/planta } C M_{P}\end{array}$ & 800,00 \\
\hline
\end{tabular}


Tabla 2. Resultados de cantidad de kg de fresa a recolectar por mes, según capacidad productiva. Fuente: autores

\begin{tabular}{|c|c|c|}
\hline \multirow[t]{6}{*}{ ETAPA VEGETATIVA } & MES 1 & 0 \\
\hline & MES 2 & 0 \\
\hline & MES 3 & 0 \\
\hline & MES 4 & 0 \\
\hline & MES 5 & 0 \\
\hline & MES 6 & 0 \\
\hline \multirow[t]{7}{*}{ ETAPA PRODUCTIVA 1} & MES 7 & 10080 \\
\hline & MES 8 & 13440 \\
\hline & MES 9 & 10080 \\
\hline & MES 10 & 9520 \\
\hline & MES 11 & 4480 \\
\hline & MES 12 & 4480 \\
\hline & MES 13 & 3920 \\
\hline \multirow[t]{2}{*}{ PODA Y RENOVACIÓN } & MES 14 & 0 \\
\hline & MES 15 & 0 \\
\hline \multirow[t]{7}{*}{ ETAPA PRODUCTIVA 2} & MES 16 & 4320 \\
\hline & MES 17 & 5760 \\
\hline & MES 18 & 4320 \\
\hline & MES 19 & 4080 \\
\hline & MES 20 & 1920 \\
\hline & MES 21 & 1920 \\
\hline & MES 22 & 1680 \\
\hline \multirow[t]{2}{*}{ PODA Y RENOVACIÓN } & MES 23 & 0 \\
\hline & MES 24 & 0 \\
\hline
\end{tabular}

Tabla 3. Costos y Función Objetivo. Fuente: Autores

\begin{tabular}{llrr}
\hline $\begin{array}{l}\text { Costo de establecimiento } \\
\text { mantenimiento (2 años) }\end{array}$ & y & $72.000 .000,00$ \\
\hline $\begin{array}{l}\text { Ventas generadas (2 años) } \\
\text { Función Objetivo (Maximizar }\end{array}$ & $\$$ & $144.000 .000,00$ \\
\hline \begin{tabular}{l} 
utilidad) \\
\hline
\end{tabular} & & $72.000 .000,00$ \\
\hline
\end{tabular}

\section{Conclusiones}

Se observa que el desarrollo del presente artículo, abarco el diseño de un modelo para la planeación de la producción de fresa; teniendo en cuenta factores de costo/beneficio en un cultivo; a partir de programación lineal, con el objetivo de identificar capacidades productivas respecto a las etapas de cultivo y su relación con la sostenibilidad esperada. Se evidencia, que el modelo a nivel teórico es funcional, permitiendo establecer un sistema de verificación de capacidades productivas, de acuerdo a parámetros de entrada, para el mismo. Así mismo, se menciona, que el presente desarrollo, representa una sección del proyecto de investigación INV_ING_1915. Se señala que a futuro se espera desarrollar el establecimiento de escenarios, respecto a posibles comportamientos en el cultivo, que permitan identificar su efectividad.

\section{Trabajos futuros}

Es necesario resaltar, que el modelo se encuentra en su primera fase de desarrollo, a partir de un contexto teórico, por tal razón se exhorta o recomienda que futuras investigaciones se enfoquen, en la comprobación y aplicación experimental con el fin de ajustar o delimitar el modelo inicial.

\section{Agradecimientos}

Los autores reconocen las contribuciones y el apoyo brindado por la Universidad Militar Nueva Granada, teniendo en cuenta que el presente artículo es un producto derivado del proyecto de investigación INV_ING_1915 "Aplicación para dispositivos móviles para el manejo de la información en un cultivo de fresa".

\section{Referencias}

[1] H. Sánchez Martin y A. Morales Garcia, «Sistema de visión artificial para la inspección, selección y control de calidad de fresas», Universidad Industrial de Santander, Bucaramanga, Colombia, 2009.

[2] L. Calderón, D. Angulo, D. Rodríguez, C. Grijalba y M. Pérez, «Evaluación de materiales para el acolchado de la fresa cultivada bajo invernadero», Universidad Militar Nueva Granada, Bogotá, Colombia, 2013.

[3] Cámara de Comercio de Bogotá, «Manual Fresa», Cámara de Comercio de Bogotá - Núcleo Ambiental S.A.S, Bogotá, Colombia, 2015.

[4] G. Herrera y L. Quintero, «Alternativas productivas para el sector agrícola de Sesquilé», Universidad del Rosario, Bogotá, Colombia, 2012.

[5] S. Rubio, A. Alfonso, C. Grijalba y M. Pérez, «Determinación de los costos de producción de la fresa cultivada a campo abierto y bajo macrotúnel», Revista colombiana de ciencias hortícolas, pp. 67-79, 2014.

[6] Chaves, Ángela, Z. Lasso, M. Ruíz y O. Benavides, «Efecto de dos coberturas plásticas y tres láminas de agua en un cultivo de fresa», Revista de ciencias agrícolas, pp. 26-37, 2013.

[7] P. Gavilán, N. Ruíz, J. Bohórquez, D. Lozano, L. Miranda y P. Domínguez, «Ahorro de agua en el cultivo de la fresa sin comprometer la producción,» Vida rural, pp. 28-33, 2015.

[8] A. M. Pastrana, «Incidencia y epidemiología de nuevos hongos patógenos de fresa en la provincia de Huelva», Universidad de Sevilla, Sevilla, 2014.

[9] A. Infante, «Producción de plantas de fresa mediante cultivo sin suelo», Horticultura, pp. 92-93, 2008.

[10] H. Alvarado , M. Tavera, G. Mena y G. Calderón , «Crecimiento y producción de fresa (fragaria x ANANASSA DUCH) en sustratos a base de compostas,» Desarrollo sustentable y finanzas, pp. 50-63, 2014.

[11] M. Bucheli, «Evaluación del impacto ambiental causado por el cultivo intensivo de fresa (Fragaria vesca) en la parroquia Huachi Granda, Cantón Ambato», Universidad Técnica de Ambato, Ambato, Ecuador 2015.

[12] K. M. Perea y O. R. Lopez, «Mejoramiento de la productividad mediante la reducción de costos en una línea manufacturera de arneses eléctricos,» Ing. USBMed, Vol. 6, No. 1, enero-junio 2015, Medellín, Colombia, 2015 